\title{
Recovering Missing Data on Satellite Images
}

\author{
Isabelle Herlin ${ }^{1,2}$, Dominique Béréziat ${ }^{3}$, and Nicolas Mercier ${ }^{1,2}$ \\ ${ }^{1}$ INRIA \\ ${ }^{2}$ CEREA, Join Laboratory ENPC-EDF R\&D - Université Paris-Est \\ ${ }^{3}$ Université Pierre et Marie Curie - LIP6
}

\begin{abstract}
Data Assimilation is commonly used in environmental sciences to improve forecasts, obtained by meteorological, oceanographic or air quality simulation models, with observation data. It aims to solve an evolution equation, describing the dynamics, and an observation equation, measuring the misfit between the state vector and the observations, to get a better knowledge of the actual system's state, named the reference. In this article, we describe how to use this technique to recover missing data and reduce noise on satellite images. The recovering process is based on assumptions on the underlying dynamics displayed by the sequence of images. This is a promising alternative to methods such as space-time interpolation. In order to better evaluate our approach, results are first quantified for an artificial noise applied on the acquisitions and then displayed for real data.
\end{abstract}

\section{Introduction}

Satellite acquisitions are commonly contaminated during the acquisition process: images display noise of various extent. Moreover, part of the data are covered by clouds. These structures are considered as occlusions in case of ocean images. The issue of recovering noisy and missing data has been extensively studied by the scientific community, in order to allow a better visualization and understanding of the information. A first class of methods groups the interpolation techniques [18. Interpolation is used to convert data acquired on an irregular grid to a regular one. B-splines are frequently chosen as they allow a good compromise between the adequacy to input data and the regularity of the result. If interpolation is applied to the issue of recovering missing data, regions can be recovered with multi-scale B-splines [10]. Another possibility is to use a normalized convolution [9] applying only on valid pixels. The kernel convolution, usually chosen Gaussian, can be driven by the local image gradient orientation [12]. However, if the surface of missing data is too large, these techniques become insufficient. A second class of methods concerns the so-called "inpainting" approaches, which make use of oriented diffusion processes. Using the local orientation of image gradient, it becomes possible to close interrupted lines [6], and even, to recover large regions by diffusing the image texture in the direction of the image gradient 4/5/7/114. However, these methods are either spatial or space-time techniques, with time only considered as an additional dimension:

A. Heyden and F. Kahl (Eds.): SCIA 2011, LNCS 6688, pp. 697-707, 2011.

(C) Springer-Verlag Berlin Heidelberg 2011 
they do not use any knowledge on the underlying dynamics that is visualized by the image sequence. In this paper, we propose an alternative and design a new data assimilation method to recover missing data, based on assumptions over the dynamics.

Section 2 briefly summarizes the weak formulation of variational data assimilation, that is applied in the paper. Section 3 describes its application to the issue of recovering missing data while Section 4 displays results and quantifies them on artificial data.

\section{Variational Data Assimilation}

Let us first summarize the principles of variational data assimilation.

\subsection{Mathematical Setting}

Let $\mathbf{X}$ being a state vector depending on the spatial location $\mathbf{x}(\mathbf{x}=(x, y)$ for $2 \mathrm{D}$ images) and time $t . \mathbf{X}$ is defined on $A=\Omega \times[0, \mathbf{T}], \Omega$ being the bounded spatial domain and $[0, \mathbf{T}]$ the temporal domain.

We assume $\mathbf{X}$ is evolving in time according to:

$$
\frac{\partial \mathbf{X}}{\partial t}(\mathbf{x}, t)+\mathbb{M}(\mathbf{X})(\mathbf{x}, t)=\mathcal{E}_{m}(\mathbf{x}, t)
$$

M, named evolution model, is supposed differentiable. As $\mathbb{M}$ describes approximately the effective evolution of the state vector, based on assumptions, a model error $\mathcal{E}_{m}$ is introduced to quantify the deviation in space and time.

Observations $\mathbf{Y}(\mathbf{x}, t)$, which are satellite image acquisitions in this paper, are available at location $\mathbf{x}$ and date $t$ and linked to the state vector through an observation equation:

$$
\mathbf{Y}(\mathbf{x}, t)=\mathbb{H}(\mathbf{X}(\mathbf{x}, t))+\mathcal{E}_{O}(\mathbf{x}, t)
$$

The observation error $\mathcal{E}_{O}$ simultaneously represents the imperfection of the observation operator $\mathbb{H}$ and the measurement errors.

We consider having some knowledge on the initial condition of the state vector at $t=0$ :

$$
\mathbf{X}(\mathbf{x}, 0)=\mathbf{X}_{b}(\mathbf{x})+\mathcal{E}_{b}(\mathbf{x})
$$

with $\mathbf{X}_{b}$ named the background value and $\mathcal{E}_{b}$ the background error.

$\mathcal{E}_{m}, \mathcal{E}_{O}$ and $\mathcal{E}_{b}$ are assumed to be Gaussian and characterized by their covariance matrices $Q, R$ and $B$ [13].

\subsection{Variational Formulation}

In order to solve the system (11), (2), (3) with respect to $\mathbf{X}$ having a maximal a posteriori probability given the observations, the functional (4) is defined and 
has to be minimized. This is called "weak formulation" of $4 \mathrm{D}$-Var, because the first term corresponds to a non perfect model.

$$
\begin{aligned}
E(\mathbf{X})= & \frac{1}{2} \int_{A}\left(\frac{\partial \mathbf{X}}{\partial t}+\mathbb{M}(\mathbf{X})\right)^{T}(\mathbf{x}, t) Q^{-1}(\mathbf{x}, t)\left(\frac{\partial \mathbf{X}}{\partial t}+\mathbb{M}(\mathbf{X})\right)(\mathbf{x}, t) d \mathbf{x} d t \\
& +\int_{A}(\mathbf{Y}-\mathbb{H}(\mathbf{X}))^{T}(\mathbf{x}, t) R^{-1}(\mathbf{x}, t)(\mathbf{Y}-\mathbb{H}(\mathbf{X}))(\mathbf{x}, t) d \mathbf{x} d t \\
& +\int_{\Omega}\left(\mathbf{X}(\mathbf{x}, 0)-\mathbf{X}_{b}(\mathbf{x})\right)^{T} B^{-1}(\mathbf{x})\left(\mathbf{X}(\mathbf{x}, 0)-\mathbf{X}_{b}(\mathbf{x})\right) d \mathbf{x}
\end{aligned}
$$

$\mathcal{E}_{m}, \mathcal{E}_{O}$ and $\mathcal{E}_{b}$ are assumed to be independent with no correlation between two space-time location and the functional $E$ represents the log-density of the joint probability law 2]. The minimization is carried out by solving the associated Euler-Lagrange equation with an auxiliary variable $\lambda$, named adjoint variable:

$$
\begin{aligned}
\lambda(\mathbf{x}, \mathbf{T}) & =0 \\
-\frac{\partial \lambda}{\partial t}+\left(\frac{\partial \mathbb{M}}{\partial \mathbf{X}}\right)^{*} \lambda & =\left(\frac{\partial \mathbb{H}}{\partial \mathbf{X}}\right)^{*}(\mathbf{x}, t) R^{-1}(\mathbf{Y}-\mathbb{H}(\mathbf{X}))(\mathbf{x}, t) \\
\mathbf{X}(\mathbf{x}, 0) & =B \lambda(\mathbf{x}, 0)+\mathbf{X}_{b}(\mathbf{x}) \\
\frac{\partial \mathbf{X}}{\partial t}+\mathbf{M}(\mathbf{X}) & =Q \lambda(\mathbf{x}, t)
\end{aligned}
$$

As the initial condition for $\lambda$ is given at time $\mathbf{T}$ (Equation (5)), $\lambda$ is computed backward in time using (6). Equation (6) makes use of two adjoint operators denoted by $\left(\frac{\partial \mathbb{M}}{\partial \mathbf{X}}\right)^{*}$ and $\left(\frac{\partial \mathbb{H}}{\partial \mathbf{X}}\right)^{*}$ that are formally the dual operators of $\frac{\partial \mathbb{M}}{\partial \mathbf{X}}$ and $\frac{\partial \mathbb{H}}{\partial \mathbf{X}}$. Solving Equations (5] not straightforward: the state vector is determined from Equations (77) and (8) using the adjoint variable and the adjoint variable is determined from Equations (5) and (6) using the state vector. To break this deadlock, an incremental method is applied, that is fully described in [3].

\section{Recovering of Missing Data}

To recover the missing data, we define the quantities described in Section 2.1 in the following way. $\mathbf{X}$ is defined as $\left(\begin{array}{ll}\mathbf{W} & \mathbf{q}\end{array}\right)^{T}: \mathbf{W}=\left(\begin{array}{ll}\mathbf{u} & \mathbf{v}\end{array}\right)^{T}$ is the motion vector, and $\mathbf{q}$ is a tracer that is compared to the image observations during the assimilation phase.

In Equation (1), $\mathbb{M}$ is equal to $\left(\mathbb{M}_{\mathbf{W}} \quad \mathbb{M}_{\mathbf{q}}\right)^{T}$, with $\mathbb{M}_{\mathbf{W}}$ and $\mathbb{M}_{\mathbf{q}}$, respectively, the evolution models of $\mathbf{W}$ and $\mathbf{q}$. A stationary assumption is used for the velocities and $\mathbb{M}_{\mathbf{W}}$ reduces to 0 . This simple heuristics is acceptable for a large range of marine processes if the velocity is less than 0.1 to 0.5 meters per second. The evolution of $\mathbf{q}$ is modeled with its transport by the velocity $\mathbf{W}$ and $\mathbb{M}_{\mathbf{q}}=\nabla \mathbf{q}^{T} \mathbf{W}$. Moreover, we assume that $\mathcal{E}_{m}$ reduces to its component on the evolution of $\mathbf{q}$. 
As the quantity $\mathbf{q}$, which is compared to the image data, is one component of $\mathbf{X}, \mathbb{H}$ is a projection and Equation (2) reduces to:

$$
I(\mathbf{x}, t)=\mathbf{q}(\mathbf{x}, t)+\mathcal{E}_{O}
$$

The variance $R$ of the Gaussian noise $\mathcal{E}_{O}$ is chosen so that $R^{-1}(\mathbf{x}, t)$ (used in Equation (2)) is almost infinitesimal, on noisy pixels. These are then discarded from the computation.

The background value $\mathbf{X}_{b}$ depends on the available knowledge. A null value is given for the background of motion $\mathbf{W}_{b}$ and the first observation is taken as background of the tracer $\mathbf{q}_{b}$.

\section{Results}

The approach, described in Section 3. is applied on satellite acquisitions and compared with state-of-the-art methods. First, an artificial noise is added to the original data, in order to quantify results. Second, our approach is used on a sequence displaying missing data, in order to illustrate its potential to recover information on satellite acquisitions.

\subsection{Artificial Noise}

A sequence of satellite Sea Surface Temperature (SST) images has been acquired by NOAA-AVHRR over the Black Sea in July 20051 (see Fig. 1).
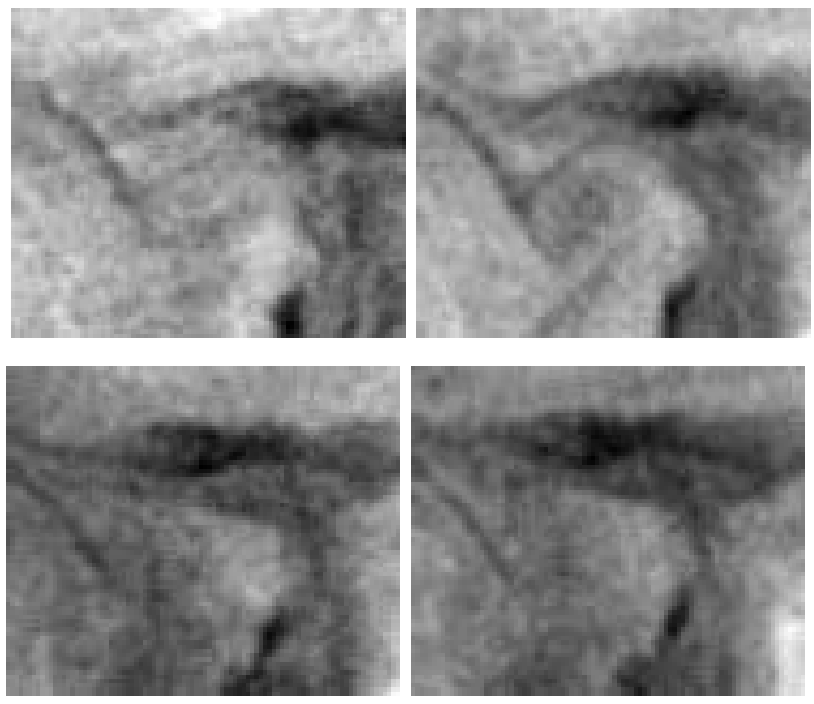

Fig. 1. NOAA-AVHRR images

${ }^{1}$ Data have been provided by E. Plotnikov and G. Korotaev from the Marine Hydrophysical Institute of Sevastopol, Ukraine. 


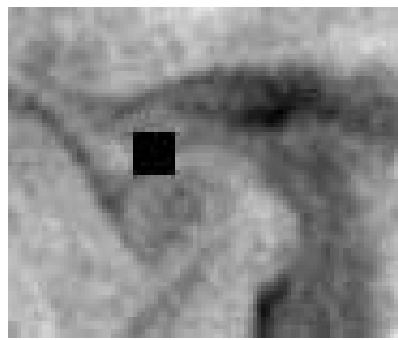

(a) Noisy image

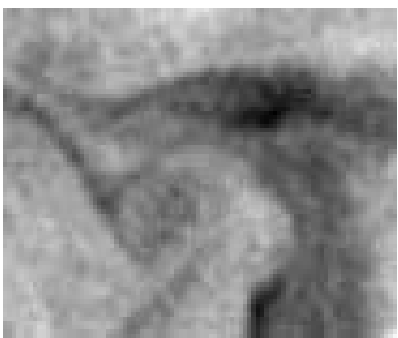

(c) Bertalmio et al

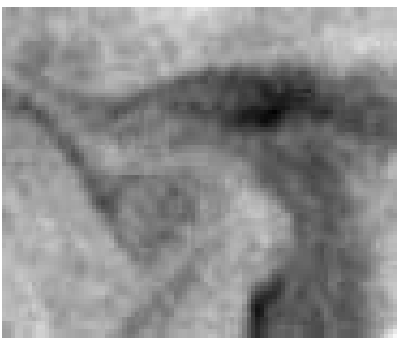

(b) Our approach

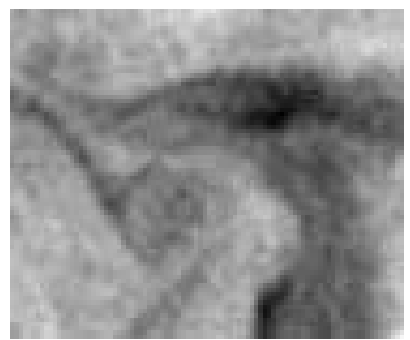

(d) Tschumperlé et al

Fig. 2. Recovering of the noisy data

In a first experiment, a noise has been added to the second image as a black square $(10 \times 10)$ (see Fig. 2). Data assimilation is then applied as explained in Section [3. Bertalmio et al [4] and Tschumperlé et al [14] are also used on the same data. Results are displayed on Fig. 2. Their quality is quantified, in Table1. by the mean, minimum and maximum of the difference between the recovered image and the original image whose grey level values over the whole sequence range from 23.428595 to 25.71952 .

In a second experiment, the noise is a $50 \times 50$ square added to the second image (see Fig. 3). The same methods are again applied and results are displayed on

Table 1. Statistics on the recovered images

\begin{tabular}{|c|ccc|}
\hline Method & Mean & Min & Max \\
\hline \hline Our approach & -0.001010 & -0.215643 & 0.382748 \\
Bertalmio et al & -0.004595 & -0.254509 & 0.145491 \\
Tschumperlé et al & -0.000339 & -0.299999 & 0.299999 \\
\hline
\end{tabular}

Table 2. Statistics on the recovered images

\begin{tabular}{|c|cccc|}
\hline Method & Mean & Min & Max & Correlation \\
\hline \hline Our approach & 0.008273 & -0.543972 & 0.769663 & 0.702 \\
Bertalmio et al & 0.023610 & -0.867842 & 0.950588 & 0.482 \\
Tschumperlé et al & 0.026362 & -0.799999 & 1.000000 & 0.572 \\
\hline
\end{tabular}




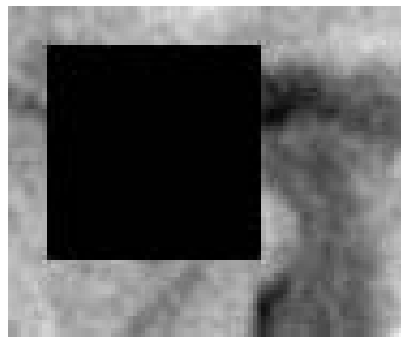

(a) Noisy image

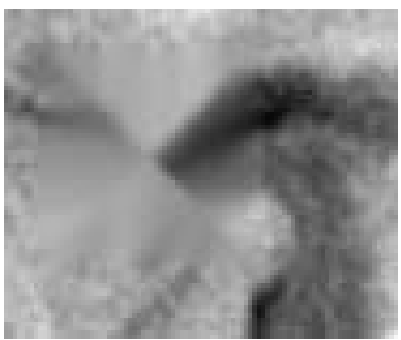

(c) Bertalmio et al

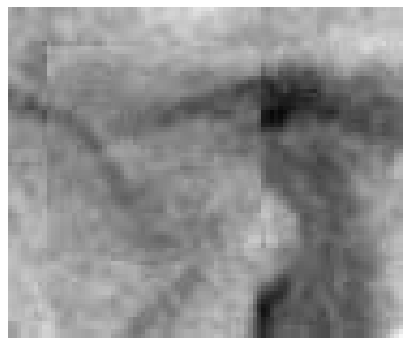

(b) Our approach

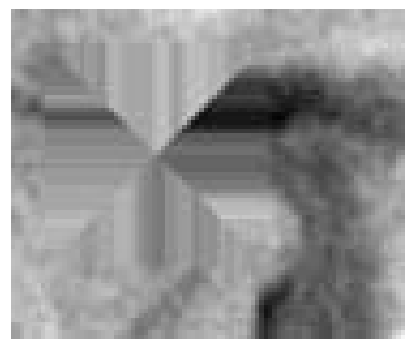

(d) Tschumperlé et al

Fig. 3. Recovering of the noisy data
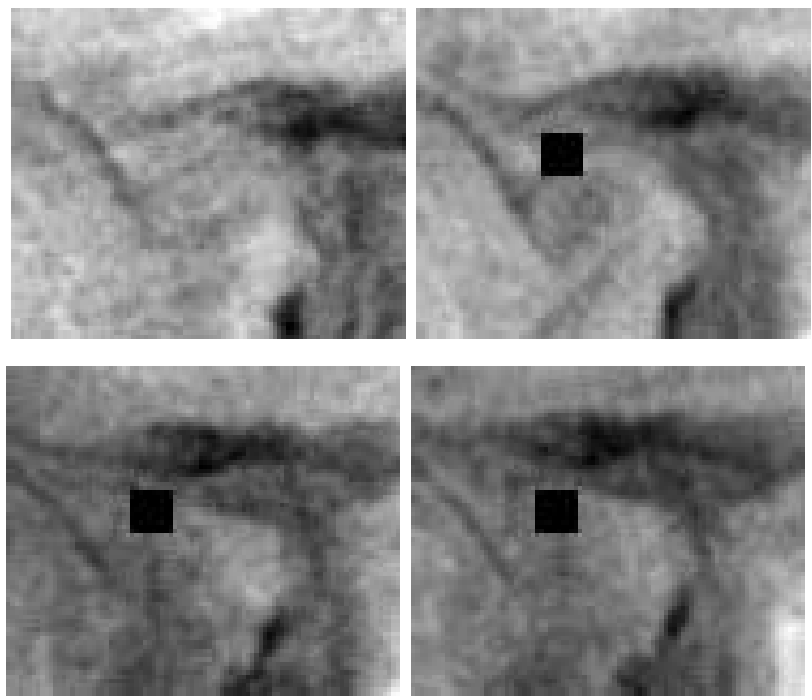

Fig. 4. Noisy sequence (squares are $10 \times 10$ )

Fig. 3. Statistics are given in Table 2, We also provide the correlation value between recovered and real data.

These experiments demonstrate that our approach is ahead of state-of-the art techniques as the size of the noisy region increases. First order statistics, correlation 

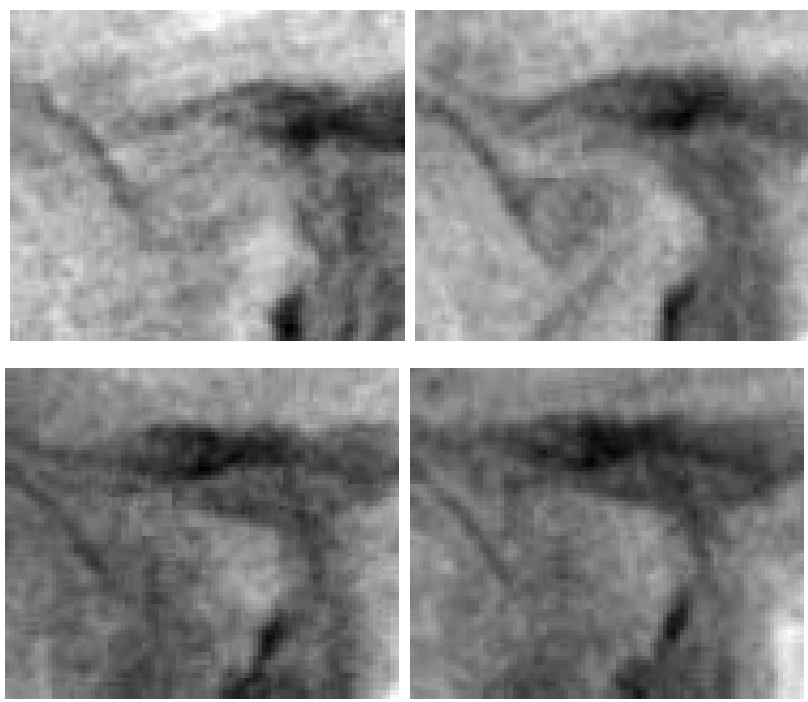

Fig. 5. Recovering of the noisy data

and visual results are much more better with our approach in the case of the $50 \times 50$ square. This demonstrates the usefulness of dynamics information in the process.

In a third experiment, noise is added on all images, except the first one (see Fig. 4). Our approach is applied and results are displayed on Fig. 5. Table 3 provides statistics (Mean, Min, Max) of the three corrupted frames, over the $10 \times 10$ squares, for the original, result and difference data.

Table 3. Error statistics

\begin{tabular}{|c|c|c|c|c|}
\hline Frame & Stat. & Original & Result & Difference \\
\hline \hline \multirow{3}{*}{2} & Mean & 24.652100 & 24.563805 & -0.001706 \\
& Min & 23.415665 & 23.415922 & -0.482744 \\
& Max & 25.715666 & 25.716354 & 0.220243 \\
\hline \multirow{3}{*}{3} & Mean & 24.652100 & 24.652100 & 0.001935 \\
& Min & 23.642063 & 23.642063 & -0.461876 \\
& Max & 26.042063 & 26.042063 & 0.151356 \\
\hline \multirow{3}{*}{4} & Mean & 24.652100 & 24.652100 & -0.00124 \\
& Min & 23.543968 & 23.543968 & -0.361067 \\
& Max & 26.143969 & 26.143969 & 0.250856 \\
\hline
\end{tabular}

These three experiments demonstrate that our approach successfully recover missing or noisy data of limited extension.

\subsection{Real Noise}

A sequence of six SST acquisitions acquired by NOAA-AVHRR over the Black Sea in May 2005 is displayed on Fig. 6. Noise is mainly due to clouds. Using 

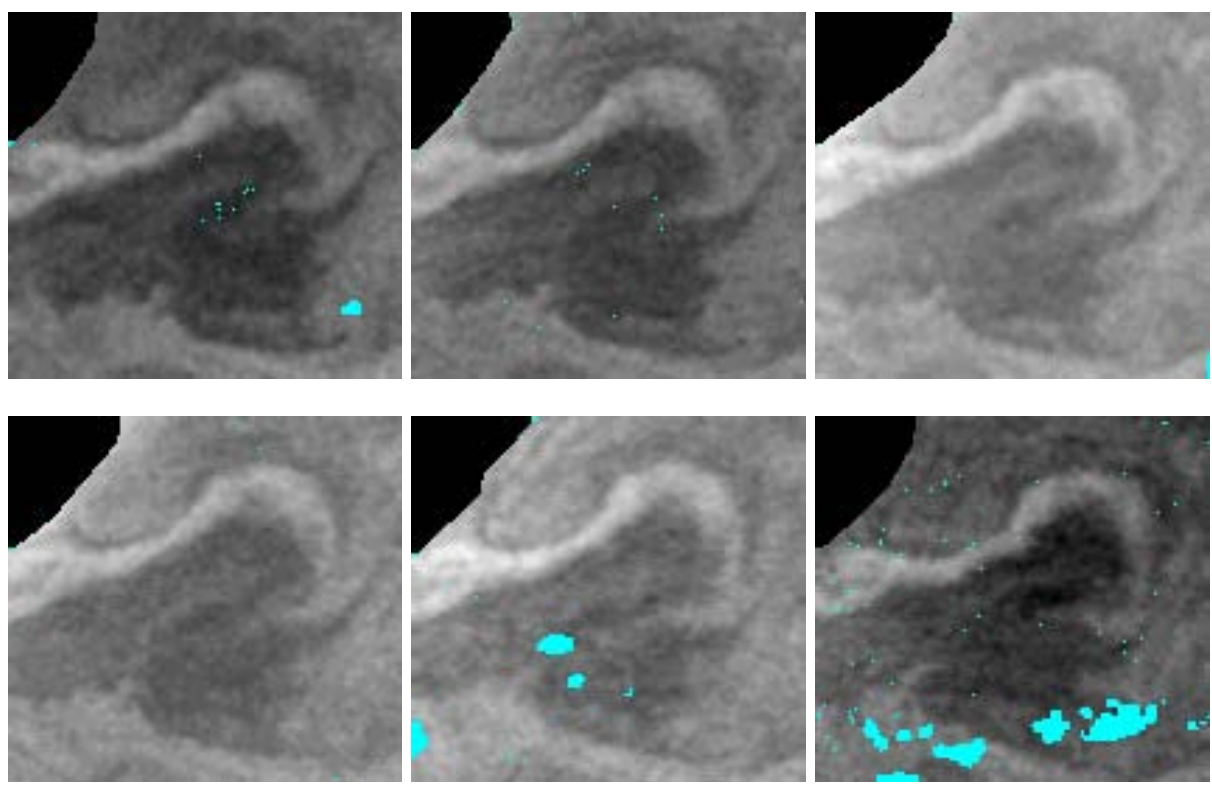

Fig. 6. Satellite acquisitions
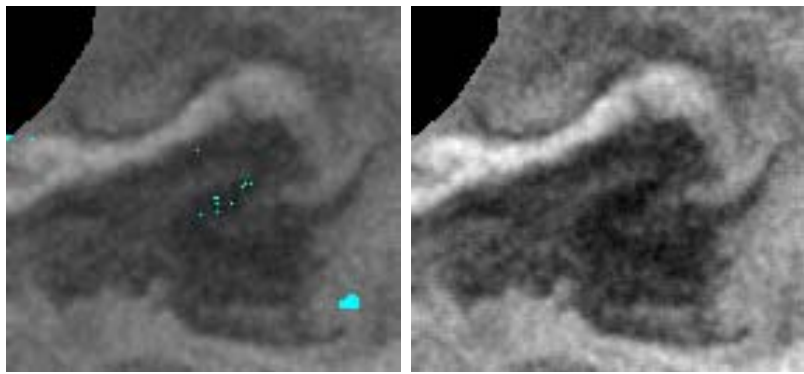

Fig. 7. First frame: original data and result of preprocessing

metadata linked to the image, a null radiometric value is given to these noisy pixels that are displayed in cyan. A specific value is given to ground pixels, which are displayed in black. This is the case for region at the left corner.

Using Bertalmio et al [4], a pre-processing is applied on the first image (see Fig. (7) in order to fill in the missing data (excepted ground pixels).

Our approach is applied. The comparison between the original and result data is displayed on Fig. 8. This demonstrates the potential of our approach. 

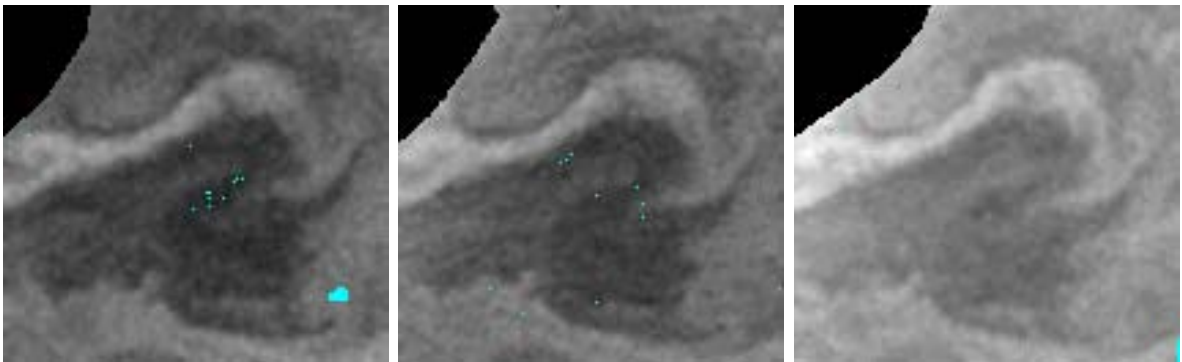

(a) Observations: frames 1, 2 and 3
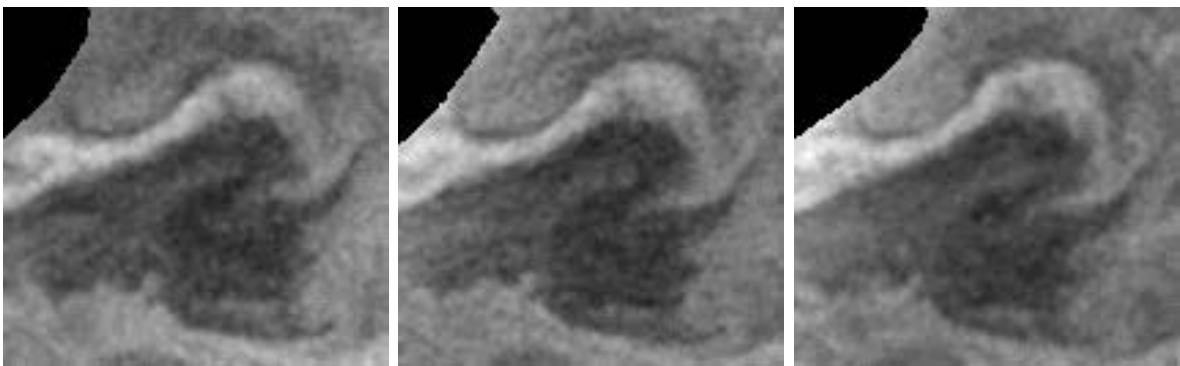

(b) Results: frames 1,2 and 3
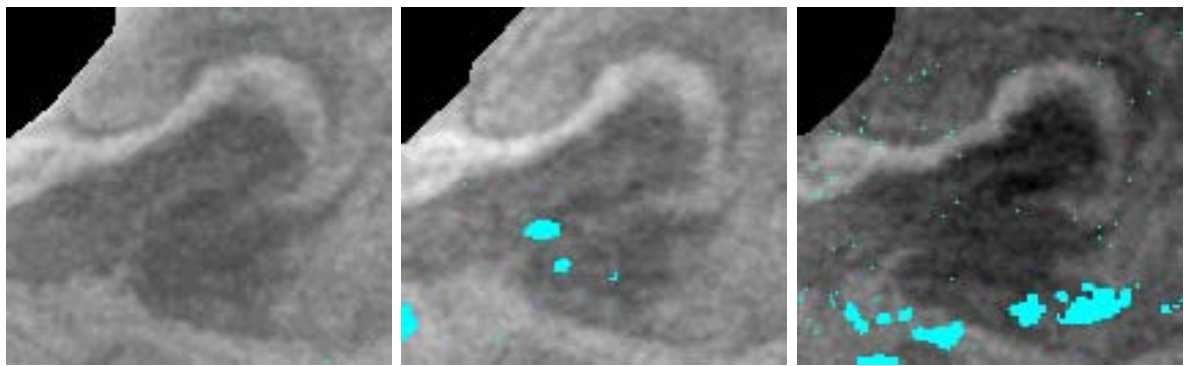

(c) Observations: frames 4, 5 and 6
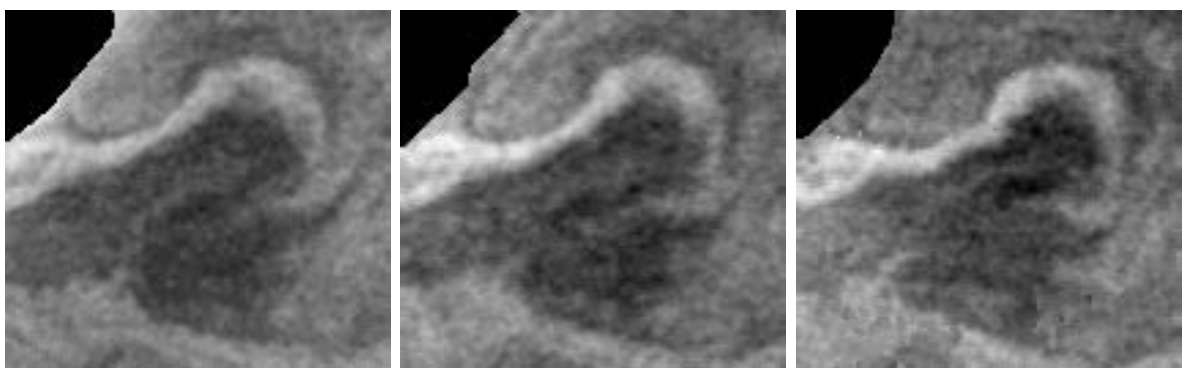

(d) Results: frames 4, 5 and 6

Fig. 8. Observations and results 


\section{Conclusion}

In this paper, we describe an approach to recover missing data on a sequence of satellite images, based on the underlying dynamics. This is an alternative to the use of spatial properties as commonly done in the state-of-the-art. The method relies on an evolution equation, describing the dynamics, and a variational data assimilation algorithm that solves the evolution equation with constraints from the observations. Given an image sequence, the data assimilation method computes a tracer $\mathbf{q}$ and a velocity field $\mathbf{W}$ on the space-time domain. The resulting $\mathbf{q}(\mathbf{x}, t)$ is the recovered image value.

We quantify the relevance of our approach on satellite data, corrupted by an artificial noise and provide comparisons with state-of-the-art methods. Our approach has also been tested on satellite images displaying natural missing data.

In order to improve the quality of results, alternative evolution equations should be considered. For instance, shallow water equations are known to correctly describe the surface velocity of SST acquisitions. Their use should allow a better recovering process. Moreover, the illumination change, due to various acquisition times over the sequence, should be better modeled in the evolution equation.

\section{References}

1. Amidor, I.: Scattered data interpolation for electronic imaging systems: a survey. Journal of Electronic Imaging 11(2), 157-176 (2002)

2. Apte, A., Jones, C.K.R.T., Stuart, A.M., Voss, J.: Data assimilation: Mathematical and statistical perspectives. Int. J. Numer. Meth. Fluids 56, 1033-1046 (2008)

3. Béréziat, D., Herlin, I.: Solving ill-posed image processing problems using data assimilation. Numerical Algorithms 52(2), 219-252 (2011)

4. Bertalmio, M., Bertozzi, A., Sapiro, G.: Navier-Stokes, Fluids Dynamics, and Image and Video Inpainting. In: Proceedings of International Conference on Computer Vision, pp. 1335-1362 (2001)

5. Bertalmío, M., Sapiro, G., Caselles, V., Ballester, C.: Image Inpainting. In: Proceedings of the International Conference on Computer Graphics and Interactive Techniques (SIGGRAPH), New Orleans, Luisiana, USA, July 23-28, pp. 417-424 (2000)

6. Breuß, M., Burgeth, B., Weickert, J.: Anisotropic continuous-scale morphology. Lecture Notes on Computer Sciences, Part II, pp. 515-522 (2007)

7. Criminisi, A., Pérez, P., Toyama, K.: Region filling and object removal by exemplarbased inpainting. Transactions on Image Processing 13(9), 1200-1212 (2004)

8. Franke, R., Nielson, G.: Scattered data interpolation and applications: A tutorial and survey. In: Geometric Modelling: Methods and Their Application, pp. 131-169. Springer, Heidelberg (1991)

9. Knutsson, H., Westin, C.-F.: Normalized and differential convolution: Methods for interpolation and filtering of incomplete and uncertain data. In: Proceedings of Conference on Computer Vision and Pattern Recognition, pp. 515-523 (1993) 
10. Lee, S., Wolberg, G., Shin, S.Y.: Scattered data interpolation with multilevel Bsplines. IEEE Transactions on Visualization and Computer Graphics 3(3), 228-244 (1997)

11. Masnou, S.: Disocclusion: a variational approach using level lines. Transactions on Image Processing 11(2), 68-76 (2002)

12. Pham, T.Q., van Vliet, L.J., Schutte, K.: Robust fusion of irregularly sampled data using adaptive normalized convolution. Journal on Applied Signal Processing, 1-12 (2006)

13. Tarantola, A.: Inverse Problem Theory and Methods for Model Parameter Estimation. Society for Industrial and Applied Mathematics, Philadelphia (2005)

14. Tschumperlé, D., Deriche, R.: Vector-Valued Image Regularization with PDE's: A Common Framework for Different Applications. In: Proceedings of Conference on Computer Vision and Pattern Recognition, pp. 651-659 (2003) 